\title{
TUMOR CARCINÓIDE DE RETO
}

\author{
PATRÍCIA ALVES MANGUEIRA ${ }^{1}$, GABRIELLA OLIVEIRA FERNANDES ${ }^{1}$, CARLÚCIO CRISTINO PRIMO $^{1}$, \\ MARCO AURÉLIO VIANA FRANÇA ${ }^{l}$, HILTON PEREIRA MAIA ${ }^{1}$, JOSÉ HERMES GOMES COSTA $^{l}$
}

\author{
${ }^{1}$ Serviço de Coloproctologia do Hospital Geral de Goiânia - Goiânia (GO), e Instituto de Coloproctologia de Goiânia - \\ Goiânia (GO) - Brasil.
}

MANGUEIRA PA, FERNANDES GO, PRIMO CC, FRANÇA MAV, MAIA HP, COSTA JHG. Tumor Carcinóide de Reto. Rev bras Coloproct, 2006;26(2):162-167.

RESUMO: OBJETIVO: estudar o tratamento e a evolução de 7 casos de tumor carcinóide de reto. PACIENTES E MÉTODO: análise retrospectiva do prontuário de 7 pacientes atendidos no Hospital Geral de Goiânia e Instituto de Coloproctologia de Goiânia. RESULTADOS: 7 casos de tumor carcinóide de reto foram diagnosticados incidentalmente durante colonoscopias realizadas por indicações diversas. Em todos os casos foram realizadas polipectomias endoscópicas. Análises histológicas e imunohistoquímicas evidenciaram tumor carcinóide em todos os casos. Realizada retossigmoidectomia anterior em dois casos, devido a comprometimento da camada muscular da mucosa e excisão local transanal em um caso, devido a evidência de neoplasia atípica. O seguimento médio foi de 28 meses com evolução satisfatória em todos os casos. CONCLUSÃO: Os 7 pacientes com tumor carcinóide de reto estudados foram inicialmente tratados com ressecção endoscópica, tendo evolução satisfatória e mantendo-se livres de doença no período de seguimento.

Descritores: Tumor carcinóide de reto, tumor neuroendócrino

\section{INTRODUÇÃO}

Os tumores carcinóides foram descritos pela primeira vez em 1888, por Lubarsch, que encontrou múltiplos tumores no íleo distal de 2 pacientes em autópsias. O termo Karzinoide foi usado por Oberndorfer em 1907 para descrever tumores ileais que tinham um comportamento mais benigno do que os adenocarcinomas típicos ${ }^{3,12,17,21,24,26,27 .}$

Fazem parte dos chamados tumores neuroendócrinos e constituem neoplasias $\operatorname{raras}^{2,}$ 6, 22, ${ }^{25}$. originadas de um precursor celular comum, as células neuroendócrinas que no trato digestivo são chamadas células de Kulchitsky e originam-se das criptas de Lieberkün 2, 4, 12, 19, 20, 21, 24, 26.
Essas células são caracterizadas histologicamente por reações positivas a sais de prata e a marcadores de tecido neuroendócrino incluindo enolase, sinaptofisina e cromogranina. ${ }^{3,26}$.

Têm a capacidade de produzir, armazenar e secretar diversos tipos de neuropeptídeos biologicamente funcionantes ou não-funcionantes ${ }^{2,12}$, ${ }^{20,25}$, sendo a serotonina a substância principal e também a mais estudada ${ }^{3,11,25}$. Essa substância é sintetizada de seu precursor, 5-hidroxitriptofano, pela enzima aromática decarboxilase ácida, que é subseqüentemente metabolizado pela monoamino-oxidase em ácido 5hidroxiindolacético (5-HIAA), o qual é secretado na urina $^{2,3}$ e pode auxiliar no diagnóstico de tumores funcionantes ${ }^{11}$. Essas células produzem outras

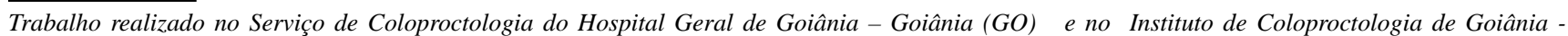
Goiânia (GO) - Brasil.

Recebido em 04/04/2006

Aceito para publicação em 08/05/2006 
substâncias, como corticotropina, histamina, dopamina, substância $\mathrm{P}$, neurotensina, prostaglandina, calicreína, entre outras ${ }^{3}$.

A Síndrome Carcinóide ocorre quando a serotonina é liberada na circulação sistêmica, causando manifestações clínicas de rubor, diarréia, broncoespasmo e eventualmente, comprometimento do coração direito ${ }^{2,3,14,20,21,25}$ quando persiste por tempo prolongado. Essa síndrome está associada a metástase hepática, na maior parte das vezes 2,25 .

Estima-se que até $50 \%$ dos pacientes com tumores carcinóides já tenham metástase ao diagnóstico ${ }^{15}$.

São tumores de crescimento lento ${ }^{2,7,15,16,19,23,}$ ${ }^{25}$, o que muitas vezes dificulta o diagnóstico em fases iniciais $^{12,20}$.

Foram classificados em 1963, por Williams e Sandler, de acordo com seu sítio embriológico de origem em Foregut (timo, pulmão, estômago, pâncreas, vesícula biliar e duodeno), Midgut (intestino delgado, apêndice e cólon direito), e Hindgut (cólon esquerdo e reto) ${ }^{1,2,3,5,12,14}$. Carcinóides originados de diferentes sítios não são apenas histologicamente e funcionalmente diferentes, mas também possuem comportamentos diferentes ${ }^{17}$.

Uma classificação mais recente, padronizada pelo WHO (World Health Organization), leva em consideração o tamanho tumoral, a invasão na parede e a presença ou não de metástases. Consiste em quatro tipos: neoplasia endócrina bem diferenciada de comportamento benigno, neoplasia endócrina bem diferenciada de comportamento incerto, carcinoma endócrino bem diferenciado (baixo grau de malignização) e carcinoma endócrino pouco diferenciado (alto grau de malignização) ${ }^{1,2,5}$.

Até o momento, não há classificação TNM para esses tumores ${ }^{1}$.

Os tumores carcinóides estão associados a uma incidência aumentada de outros tumores malignos, especialmente do trato gastrointestinal, independentemente do seu sítio de origem. Uma possível razão para o aumento na incidência de outros cânceres poderiam ser as propriedades tumorigênicas dos peptídeos secretados pelas células neuroendócrinas ${ }^{24}$.

Os carcinóides de reto foram descritos pela primeira vez em 1912 e correspondem a 1-2\% de todos os tumores de reto ${ }^{1,2,20}$. Em contraste com os carcinóides de intestino delgado e cólon, que produzem mais serotonina, produzem principalmente glucagon e peptídeos de glicetina ${ }^{2,3}$. Caracteristicamente, a grande maioria é não-funcionante ${ }^{20}$.

Cinqüenta por cento dos tumores carcinóides de reto são assintomáticos e diagnosticados incidentalmente, por endoscopias feitas por indicações diversas ${ }^{10,20,21,24}$ e a síndrome carcinóide é rara, apesar de potencialmente metastatizante ${ }^{8,20}$.

Aproximadamente $10 \%$ dos carcinóides retais podem estar associados a outra neoplasia colônica ${ }^{2}$. O tumor carcinóide apresenta-se como lesão polipóide, séssil, endurecida, móvel, de localização submucosa e de aspecto amarelado, ao exame macroscópico, o que denota alto teor lipídico ${ }^{20,21,24}$ (Figura -1). O potencial de malignização e o prognóstico dos tumores carcinóides de reto têm sido intimamente relacionados com o seu tamanho ${ }^{2}$. As metástases nesses tumores ocorrem principalmente para fígado e linfonodos regionais ${ }^{10,18}$

A invasão ou não da muscular da mucosa determina se a excisão endoscópica é suficiente ou se é necessária complementação cirúrgica nos casos de carcinóides retais ressecados endoscopicamente. Recentemente, aceita-se que lesões menores que $1 \mathrm{~cm}$ de diâmetro podem ser adequadamente tratadas por ressecção endoscópica se margens negativas após avaliação histológica forem obtidas ${ }^{10,12,21}$. Outra abordagem seria a excisão local transanal, uma vez que $75 \%$ dos tumores retais se localizam nos terços médio e inferior do reto ${ }^{1,3,20}$.

O tratamento de pacientes com tumores maiores que $2 \mathrm{~cm}$ de diâmetro consiste na ressecção

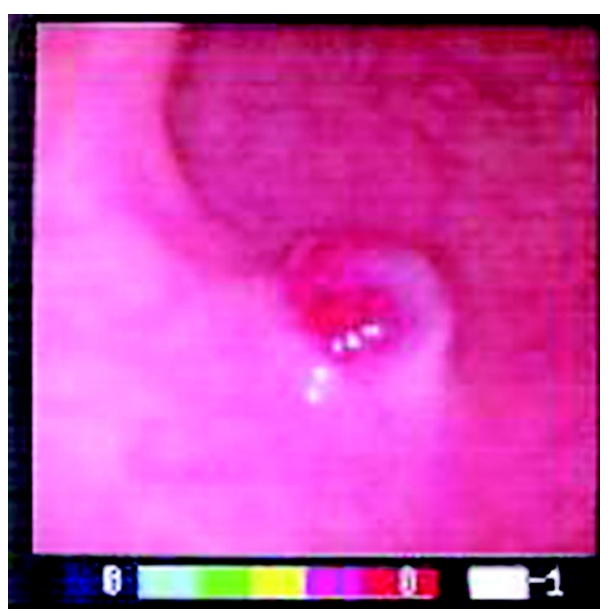

Figura 1 - Tumor carcinóide de reto. 
abdominal anterior ou amputação abdominal de reto ${ }^{1,}$ ${ }^{20}$. Entretanto, o valor desses procedimentos no tratamento de carcinóides retais tem sido recentemente questionado, uma vez que parece não aumentar a sobrevida com relação àquela observada com a excisão local, em estudos restrospectivos ${ }^{3,24}$.

A conduta em tumores de $1-2 \mathrm{~cm}$ de diâmetro é controversa. Alguns autores sugerem que pacientes com ulceração tumoral e invasão da muscular da mucosa devem ser submetidos à cirurgia radical, visto que esses fatores seriam de pior prognóstico ${ }^{3,20}$.

A taxa de sobrevida em 05 anos é de $81 \%$ para pacientes com doença localizada, $47 \%$ para pacientes com doença regional e $18 \%$ para pacientes com metástase à distância² .

\section{OBJETIVO}

Avaliar o tratamento e a evolução de 07 casos de tumor carcinóide de reto de até $2 \mathrm{~cm}$ de diâmetro, diagnosticados no Instituto de Coloproctologia de Goiânia e Hospital Geral de Goiânia.

\section{PACIENTES E MÉTODO}

Análise retrospectiva do prontuário de 07 pacientes acompanhados no Instituto de Coloproctologia de Goiânia e no Hospital Geral de Goiânia, no período de abril de 1998 a abril de 2005.

\section{RESULTADOS}

Foram estudados 3 homens e 4 mulheres com idade média de 53 anos (33-70). Quadro-1.

A maioria dos pacientes procurou o serviço com queixas decorrentes de doença colorretal orificial e o diagnóstico de tumor carcinóide foi feito incidentalmente através de colonoscopia por causas diversas. Uma paciente era portadora de retocolite ulcerativa.

O tamanho tumoral variou de 0,8 a $2,0 \mathrm{~cm}$ de diâmetro e em todos os casos foi feita ressecção endoscópica.

O exame anátomo-patológico das lesões diagnosticou tumor carcinóide de reto em todos os

\begin{tabular}{|c|c|c|c|c|c|c|c|c|}
\hline CASO & SEXO & IDADE & QUEIXA & PÓLIPO & $\begin{array}{l}\text { ANÁTOMO- } \\
\text { PATOLÓGICO }\end{array}$ & $\begin{array}{l}\text { IMUNO- } \\
\text { HISTOQUÍMICA }\end{array}$ & $\begin{array}{l}\text { OUTROS } \\
\text { TRATAMENTOS }\end{array}$ & $\begin{array}{l}\text { SEGUI- } \\
\text { MENTO }\end{array}$ \\
\hline 01 & $\mathrm{~F}^{*}$ & 54 & Sangramento & $2,0 \mathrm{~cm}$ & Carcinóide & Carcinóide & $\begin{array}{l}\text { Retossigmoi- } \\
\text { dectomia Anterior }\end{array}$ & 84 meses \\
\hline 02 & $\mathbf{M}^{\dagger}$ & 51 & $\begin{array}{l}\text { Sangramento } \\
\text { Fístula }\end{array}$ & $1,0 \mathrm{~cm}$ & Carcinóide & Não realizado & $\longrightarrow$ & 48 meses \\
\hline $\mathbf{0 3}$ & $\mathrm{F}^{*}$ & 70 & $\begin{array}{l}\text { Controle } \\
\text { RCUI }^{\ddagger}\end{array}$ & $1,5 \mathrm{~cm}$ & Carcinóide & Carcinóide & 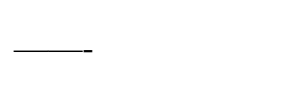 & 36 meses \\
\hline 04 & $\mathbf{M}^{\dagger}$ & 44 & Sangramento & $1,0 \mathrm{~cm}$ & Carcinóide & Carcinóide & $\longrightarrow$ & 12 meses \\
\hline 05 & $\mathbf{M}^{\dagger}$ & 66 & Sangramento & $2,0 \mathrm{~cm}$ & $\begin{array}{l}\text { Adenocar- } \\
\text { cinoma 'de novo' }\end{array}$ & Carcinóide & $\begin{array}{l}\text { Retossigmoi- } \\
\text { dectomia Anterior }\end{array}$ & 12 meses \\
\hline 06 & $\mathrm{~F}^{*}$ & 33 & $\begin{array}{l}\text { Prurido } \\
\text { Anal }\end{array}$ & $0,8-1,0 \mathrm{~cm}$ & $\begin{array}{l}\text { Carcinóde } \\
\text { atípico }\end{array}$ & $\begin{array}{l}\text { Carcinóide } \\
\text { Atípico }\end{array}$ & Ressecção Local & 05 meses \\
\hline 07 & $\mathrm{~F}^{*}$ & 63 & $\begin{array}{l}\text { Obstipação } \\
\text { Crônica }\end{array}$ & $1,5 \mathrm{~cm}$ & Carcinóide & Carcinóide & - & 05 meses \\
\hline
\end{tabular}

Quadro 1 - Distribuição dos casos estudados por sexo, idade, indicação endoscópica, tamanho da lesão tumoral, exames e tratamentos realizados, período de seguimento. $\quad *$ Feminino; $\dagger$ Masculino; $\ddagger$ Retocolite Ulcerativa Idiopática. 
casos, com exceção de um em que houve dúvida entre adenocarcinoma "de novo" ou tumor neuroendócrino. Em um dos casos estudados, o da paciente mais jovem, o exame anátomo-patológico diagnosticou tumor carcinóide atípico.

Em 6 dos 7 casos foi realizado estudo imunohistoquímico que confirmou o diagnóstico de tumor carcinóide de reto.

Em dois pacientes as lesões apresentavam $2,0 \mathrm{~cm}$ de diâmetro e a histologia mostrou invasão da muscular da mucosa. Ambos foram submetidos a retossigmoidectomia anterior para complementação do tratamento. A avaliação histológica das peças cirúrgicas não mostrou doença residual.

A paciente mais jovem cujo diagnóstico foi tumor carcinóide atípico, foi submetida a ressecção local transanal, além da polipectomia, e o exame anátomo-patológico da peça cirúrgica também não mostrou doença residual.

Todos os pacientes foram seguidos com exame colonoscópico de controle, com freqüência variável de acordo com o cirurgião. Esses exames foram normais durante o período de seguimento, com exceção de uma paciente, que havia sido submetida a retossigmoidectomia anterior cujo exame mostrou pólipo em cólon transverso e o exame anátomopatológico da lesão revelou pólipo hiperplásico.

Todos os pacientes permaneceram sem doença no período de seguimento, que foi, em média, de 28 meses.



Figura 2 - Tumor carcinóide de reto.

\section{DISCUSSÃO}

A distribuição por sexos e por faixa etária em nosso estudo está de acordo com a literatura que mostra predomínio dos tumores neuroendócrinos no sexo feminino $^{4,14,17}$ e faixa etária mais atingida em torno da sexta década de vida ${ }^{3}$; dentre nossos pacientes, 4 eram mulheres e 3 eram homens, e a média de idade foi de 53 anos.

Todos os pacientes estudados eram assintomáticos e foram diagnosticados incidentalmente, por exames colonoscópicos solicitados devido a outras patologias. Na literatura, estima-se que o diagnóstico incidental ocorra em torno de $50 \%$ dos casos para carcinóides de reto ${ }^{10,20}$. Os pacientes que têm sintomas geralmente apresentam sangramento retal, dor, ou constipação ${ }^{8}$. Uma de nossas pacientes apresentava manifestações de retocolite ulcerativa. Há relatos de uma associação aumentada entre o tumor carcinóide de reto e essa doença inflamatória ${ }^{24}$.

Todos os pacientes foram submetidos a polipectomia e a exame histopatológico, que é o determinante do diagnóstico (Figuras-2 e 3). Atualmente faz-se confirmação por estudo imunohistoquímico, através da pesquisa dos marcadores tumorais de células neuroendócrinas,

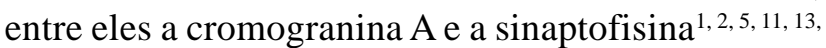
${ }^{25}$. Neste estudo, apenas um caso não foi submetido ao estudo imunohistoquímico, devido à amostra ser insuficiente.

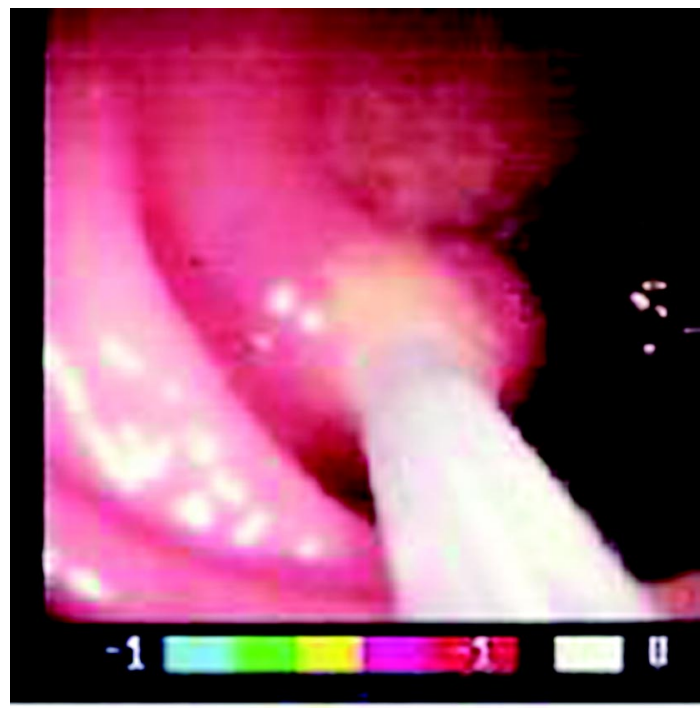

Figura 3 - Polipectomia endoscópica. 
Nos dois casos em que os diâmetros tumorais foram de $2,0 \mathrm{~cm}$, houve comprometimento de muscular da mucosa e os pacientes necessitaram de tratamento adicional com retossigmoidectomia, uma vez que há íntima relação entre o tamanho tumoral e a presença de metástases ${ }^{2,10,18}$. Elas ocorrem em aproximadamente $2 \%$ dos pacientes com lesões menores que $1 \mathrm{~cm}$ de diâmetro, enquanto lesões entre $1-2 \mathrm{~cm}$ apresentam metástases em 10-15\% dos casos. Pacientes com tumores maiores que $2 \mathrm{~cm}$ freqüentemente apresentam metástases em 60-80\% dos casos ${ }^{2,3,20}$.

Uma das pacientes estudadas apresentou carcinóide atípico e foi submetida a excisão local. Há controvérsias na literatura sobre o conceito de tumor "atípico", mas é consenso que este possui mais de 2 mitoses $/ \mathrm{mm}^{2}$ e mais de $2 \%$ de células Ki-67+ (status proliferativo), sugerindo um comportamento mais agressivo nesse tipo tumoral ${ }^{20}$, em contraste com a maioria dos tumores neuroendócrinos, que possuem monomorfismo celular com ausência ou baixo grau de atipia ( $<2$ mitoses/ $\mathrm{mm} 2$ ) e baixo status proliferativo $(<2 \%$ de células Ki-67+), indicando um crescimento lento e comportamento benigno ${ }^{1,2,26}$. Essa paciente não necessitou de procedimento adicional, uma vez que não apresentou invasão de muscular da mucosa ao exame histológico.

\section{CONCLUSÃO}

Os 7 pacientes do presente estudo que, submetidos a colonoscopia por indicações diversas, tiveram diagnóstico incidental de tumor carcinóide de reto menores que $2 \mathrm{~cm}$ de diâmetro, foram todos primeiramente tratados com ressecção endoscópica, em concordância com a literatura, tendo evolução satisfatória e mantendo-se livres da doença no período de seguimento.

SUMMARY: OBJECTIVE: The aim of the present study was to analyze the treatment and follow-up of seven patients who suffered from rectal carcinoid tumor. PATIENTS AND METHOD: The authors have reviewed the records of seven patients operated on at the Hospital Geral de Goiânia and at the Instituto de Coloproctologia de Goiânia. RESULTS: Seven cases of rectal carcinoid tumors were accidentally found during full colonoscopy assesment due to varied complaints. All of them were treated with endoscopic polipectomy and microscopy and immunochemistry have confirmed carcinoid tumor. Two patients underwent anterior resection due to invasion of the muscularis mucosae and one patient underwent transanal local excision due to atypical changes. The average follow-up time was 28 months and all the patients had an uneventful postoperative course.

Key words: Rectal carcinoid tumor; Neuroendocrine tumor.

\section{REFERÊNCIAS BIBLIOGRÁFICAS}

1. Ramage JK, Davies AHG, Ardill J, Bax N, Caplin M, Grossman A. et al. Guidelines for the management of gastroenteropancreatic neuroendocrine (including carcinoid) tumours. Gut. 2005; 54 (IV):1-16.

2. Kaltsas GA, Besser GM, Grossman AB. The diagnosis and medical management of advanced neuroendocrine tumors. Endocrine Reviews 2004; 25(3): 458-511.

3. Kulke M, Mayer RJ. Medical Progress: Carcinoid Tumors. N Engl J Med. 1999; 340 (11): 858-868.

4. Maggard MA, O'Connell JB, Ko CY.Update populationbased review of carcinoid tumors. Ann Surg. 2004; 240(1): 117-122.

5. Öberg K. Carcinoid Tumors: molecular genetics, tumor biology, and update of diagnosis and treatment. Curr Opin Oncol.2002; 14(1): 38-45.
6. Öberg K. Neuroendocrine tumors of the gastrointestinal tract: recent advances in molecular genetics, diagnosis, and treatment. Curr Opin Oncol.2005; 17(4): 386-391.

7. Pelley RJ, Bukowski RM. Recent advances in systemic therapy for gastrointestinal neuroendocrine tumors. Curr Opin Oncol.1999; 11(1):32.

8. Maroy B. Similar Rectal Carcinoid Tumors of two sibilings with curative endoscopic snare resection. J Clin Gastroenterol. 1997; 24(2): 124-125.

9. Sharma R, McLeod D, Clarke SJ. Small blue cell tumors of the rectum: Case 3. Atypical carcinoid of the rectum. J Clin Oncol. 2005; 23(4): 914-915.

10. Banzo J, Vidal-Sicat S, Prats E, Galofré G, Razola P, Mañé S et al. In-111 DTPA octreotide scintigraphy and intraoperative gamma probe detection in the diagnosis and treatment of residual lymph node metastases of a rectal carcinoid tumor. Clin Nucl Med. 2005; 30(5) 308-311. 
11. Taupenot L, Harper KL, O'Connor DT. Mechanisms of disease: the chromogranin-secretogranin family. N Engl J Med. 2003; 348(12): 1134-1149.

12. Shebani KO, Souba WW, Finkelstein DM, Stark PC, Elgadi $\mathrm{KM}$, Tanabe KK et al. Prognosis and survival in patients with gastrointestinal tract carcinoids tumors. Ann Surg. 1999; 229(6): 815

13. Erickson LA, Lloyd RV. Practical markers used in the diagnosis of endocrine tumors. Adv Anat Pathol 2004. 11(4): 175-189.

14. Dierdorf SF. Carcinoid tumor and carcinoid syndrome. Curr Opin Anaesthesiol 2003; 16(3): 343-347.

15. Jensen RT. Carcinoid and pancreatic endocrine tumors: recent advances in molecular pathogenenesis, localization and treatment. Curr Opin Oncol 2000; 12(4): 368-377.

16. Cheng JY, Sheu LF, Meng CL, Lin JC. Expression of p53 in colorectal carcinoids. Arch Surg. 1996; 131(1): 67-70.

17. Onaits MW, Kirshbom PM, Hayard TZ, Quayle FJ, Feldman JM, Seigler HF et al. Gastrointestinal carcinoids: characterization by site of origin and hormone production. Ann Surg. 2000; 232(4): 549-556.

18. Venook AP. Embolization and chemoembolization therapy for neuroendocrine tumors. Curr Opin Oncol. 1999; 11(1): 38.

19. Barakat MT, Meeran K. Neuroendocrine tumors and gut hormones. Curr Opin Endocrinol Diabet 2005; 12(1): 99-105.

20. Rossi BM, Nakagawa WT, Ferreira FO, Aguiar Jr S, Lopes A . Câncer de Cólon, Reto e Ânus. Ed Marina e Ed Tecmedd. 2005; 40: 535-538.

21. Maizier WP, Levien DH, Luchtefeld MA, Senagore AJ. Surgery of the Colon, Rectum, and Anus. W. B. Saunders Company. 1995; 47: 544-545.
22. Boudreax JP, Putty B, Frey DJ, Woltering E, Anthony L, Dally I et al. Surgical treatment of advanced-stage carcinoid tumors: lessons learned. Ann Surg. 2005; 241(6): 839-846.

23. Kwekkeboom DJ, Teunissen JJ, Bakker WH, Kooij PP, de Heder WW, Feelders RA et al. Radiolabeled somatostatin analog [177Lu-DOTAo, Tyr3] octreotate in patients with endocrine gastroenteropancreatic tumors. J Clin Oncol.2005.23(12): 2754-2762.

24. Corman ML: Colon \& Rectal Surgery. Philadelphia.5a. Ed. Lippincott Williams \& Wilkins, 2005.p.1091-1099.

25. Lal A, Chen H. Treatment of advanced carcinoid tumors. Curr Opin Oncol. 2006; 18(1): 9-15.

26. Fenwick SW, Wiatt JI, Toogood GJ, Lodge JPA. Hepatic resection and transplantation for primary carcinoid tumors of the liver. Ann Surg. 2004; 239 (2): 210-219.

27. Yantiss RK, Odze RD, Farraye F, Rosenberg AE. Solitary versus multiple carcinoid tumors of the ileum: a clinical and pathologic review of 68 cases. Am J Pathol. 2003; 27(6): 811817.

\section{Endereço para correspondência:} PATRÍCIA ALVES MANGUEIRA

Av: C n. 1129 - Quadra 41 - Lote 38 - Setor Couto Magalhães 77.824-780 - Araguaina (TO) 